\title{
Detection of scattered light in telescopes
}

\author{
F. Grundahl and A.N. Sørensen \\ Institute of Physics and Astronomy, University of Aarhus, DK-8000 Århus C, Denmark
}

Received August 29; accepted September 25, 1995

\begin{abstract}
We present a simple, but powerful method to test for the presence of scattered light in telescopes. Scattered light can seriously hamper any attempt to achieve a proper flat fielding of CCD data. The method consists of making a pinhole camera (camera obscura) by placing a pinhole mask in front of the CCD window. Exposing the CCD in this configuration gives an image of the light sources in the optical path. Tests made at the Nordic Optical Telescope have led to a reduction of large scale flat field variations due to scattered light from a total of $\sim 5 \%$ to below $1 \%$ by improving the telescope baffles.
\end{abstract}

Key words: instruments: miscellaneous - telescopes

\section{Introduction}

An essential ingredient in most applications of charge coupled devices (CCD's) is flat fielding, which corrects for the spatially varying sensitivity across the CCD surface. The way to obtain a 'correct' flat field is still a matter of debate, even though it is rarely discussed in the literature. For a few examples of the approaches adopted by different authors the reader is referred to the papers by Stetson \& Harris (1988), Penny \& Griffiths (1991) and Kuhn et al. (1991).

Most often the flat field is obtained by exposing on an evenly illuminated screen in the dome, or the twilight sky. This approach is usually adequate for obtaining the pixelto-pixel variations with sufficient precision. However, this may not be the case for the large scale variations of the flat field as we will show later.

Flat field problems may be a part of the explanation of the fact that CCD photometry has only in a few cases obtained an accuracy comparable to that obtained by photoelectric photometry. It is well known that very high precision may be obtained in differential time series photometry using CCD's. However in these applications the stars under study are nearly always placed very near the same positions on the CCD (Kjeldsen \& Frandsen 1992; Gilliland et al. 1993), and no attempt to transform to a standard system is made.

Often it is possible to obtain quotients of dome or sky flat fields which are stable at the $1 \%$ level. But this is not necessarily the precision of the flat fields. Tobin (1992) finds that although quotients of dome and sky flat fields reproduce to better than $1 \%$, the results from doing pho-

Send offprint requests to: F. Grundahl; fgj@obs.aau.dk tometry of a close double star depend on its position in the CCD frame, and the intensity ratio varies by up to $4 \%$. The source of this error is identified by Tobin to be scattered light reaching the CCD. Garnavich et al. (1994) has also reported a problem in their photometry of NGC 6791 caused by scattered light.

The quality of the obtained flat fielding of science images is sometimes judged by the flatness of the sky background. If scattered light is present, it may be dangerous to assume that a flat sky background also indicates a 'perfect' flat fielding. See Andersen et al. (1995).

The motivation behind the present paper comes from flat field problems encountered with data obtained at the $2.56 \mathrm{~m}$ Nordic Optical Telescope (NOT) on La Palma during several observing runs. The NOT is an alt-az mounted telescope and thus it has a field de-rotator to counter the effects of the rotating Earth. This causes the orientation of the CCD camera to change relative to the telescope during tracking. If sources other than the secondary mirror contribute to the light seen at the position of the CCD they can cause the flat field exposures to depend on rotator position.

This was one of the problems experienced by several observers at NOT. Flat fields (dome and twillight sky flat fields) obtained at different rotator positions showed quite large differences. Quotients of dome flat fields obtained at rotator positions of, say, $90^{\circ}$ and $180^{\circ}$ showed total variations (primarily as a gradient) of typically $5 \%$ in broad band filters. A variation of this magnitude obviously represents a serious problem if calibrated photometry is to be done. A variation of a similar size was also seen in quotients between sky and dome flats. As we shall show 


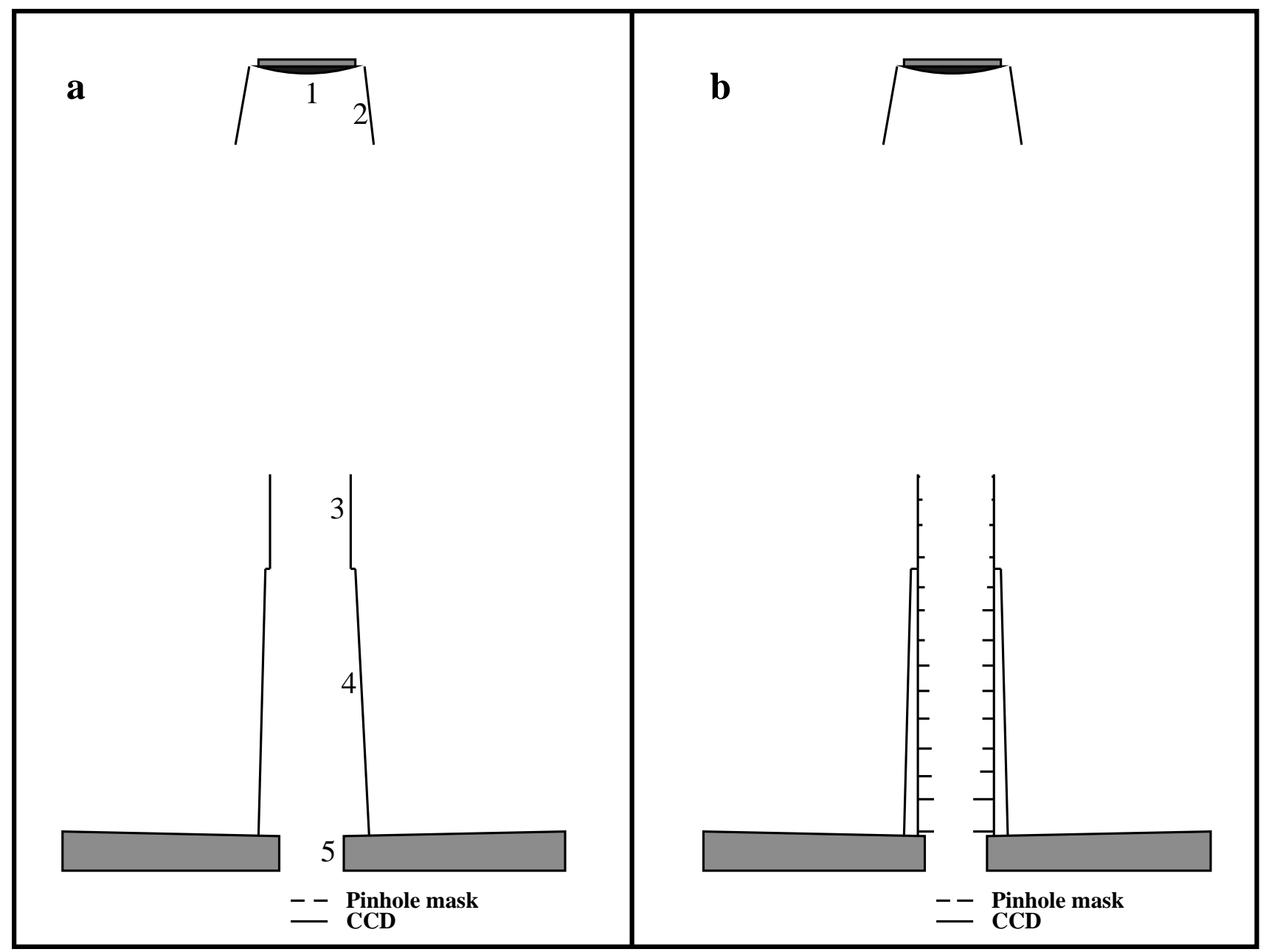

Fig. 1. The optical system of NOT before and after the baffle modification. a) The telescope baffles before installation of the extra baffles. The regions labelled correspond to: 1) the secondary mirror, 2) the inside of the secondary mirror baffle, 3 ) the upper end of the primary mirror baffle, 4) the lower end of the primary mirror baffle and 5) a metal collar located in the Cassegrain hole. The collar is used for supporting the primary mirror baffle. b) Same as a), but with the baffle modification installed

below this variation is caused by scattered light in the optical system.

Here we describe a simple method to identify sources of scattered light that may be present in the optical path of a telescope (equatorially or alt-az. mounted). We also describe actions taken at NOT to reduce the scattered light, and the consequences for the precision of the flat fields obtained.

\section{The pinhole camera}

The simplest test for identifying scattered light is to remove the CCD camera and place the naked eye at its position. We did this test at NOT, and it clearly revealed reflections from the inside of the primary mirror baffle. But how could we determine if they had a significant contribution to the flat field problems we had encountered?
By placing a piece of cardboard with a pinhole in it in front of the CCD, we made a 'camera obscura' which would show the inside of the telescope as seen from the CCD. Obtaining CCD images in this configuration allowed us to give a quantitative estimate of the sources of scattered light.

We here discuss pinhole images obtained in September 1993 at NOT, just prior to and just after a modification of the main mirror baffle.

The images were obtained with exactly the same configuration as the one used when doing dome flats, except that a pinhole mask was put in front of the CCD. The CCD camera used a $2048 \times 2048$ pixel Loral chip, mounted in the Cassegrain focus. An on-chip binning factor of 4 was used. The total field of view of the pinhole camera was $\sim 22 \mathrm{deg}$. 


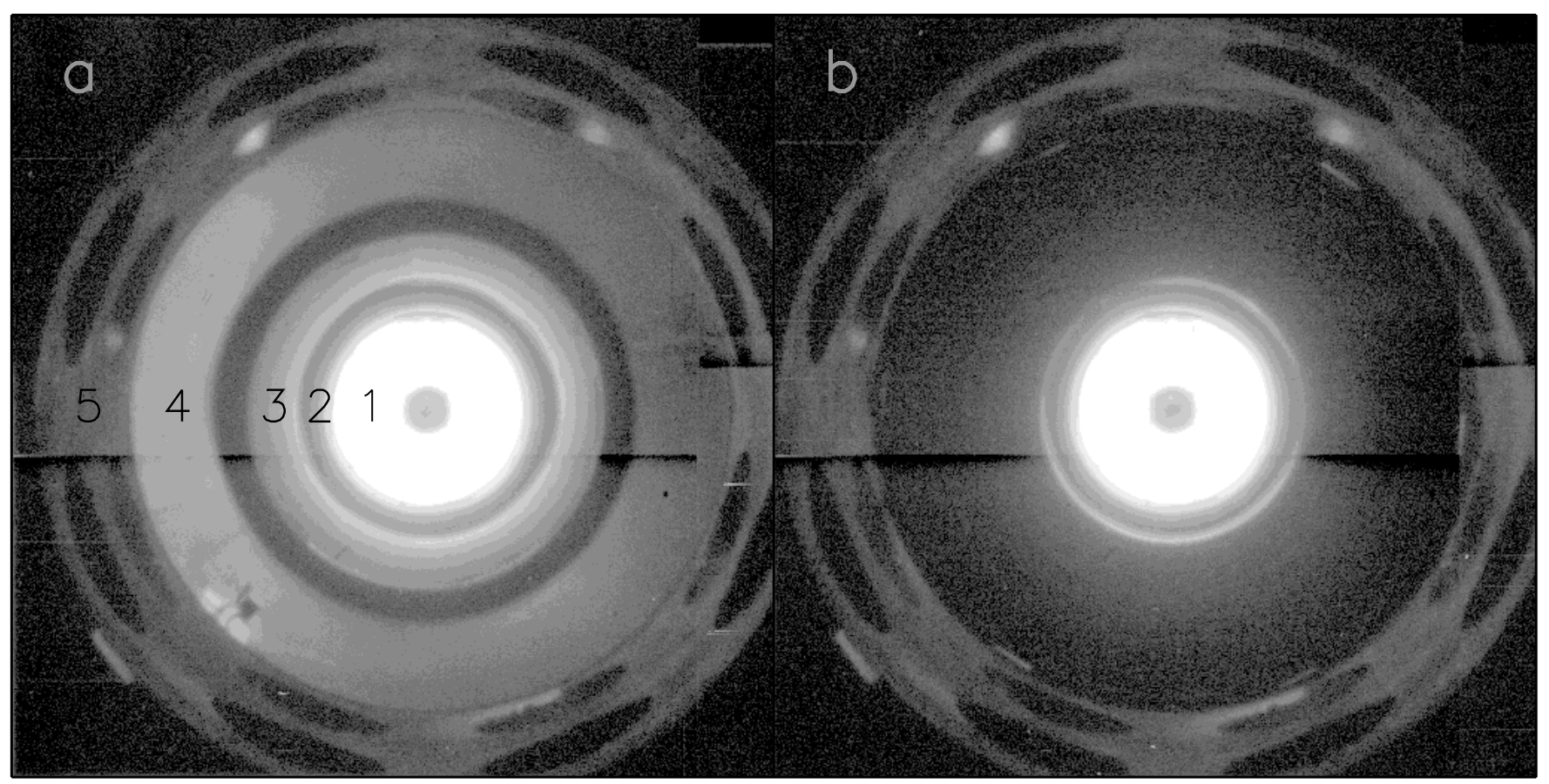

Fig. 2. The pinhole images obtained before and after the baffle modification. Both are logarithmically scaled. a) Before modification. The numbers shown correspond to light from the areas labelled in Fig. 1a. b) Note the dramatic improvement after the baffle modification. Each of the images is a combination of two images taken at two rotator angles, and the horizontal line below the secondary mirror is caused by an area with a number of bad coloumns

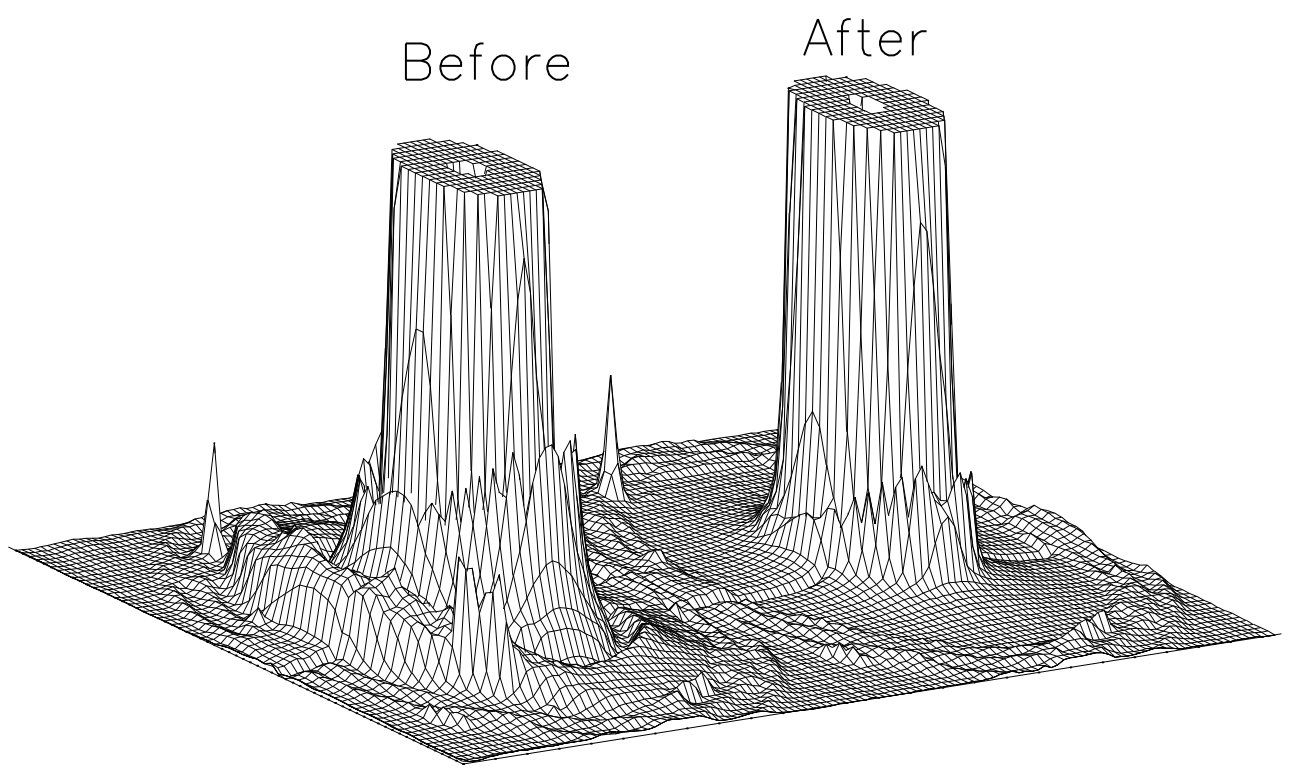

Fig. 3. Surface plot of the pinhole images. It is obvious from this plot, how the light distribution was strongly asymmetric before the baffle modification. Note that the scale in this plot is linear. The spikes are are caused by light from bulbs in the dome passing through holes in the collar in the Cassegrain hole. The peak level in this plot has been cut to 2500 ADU for display purposes, it is $20000 \mathrm{ADU}$ in the images 

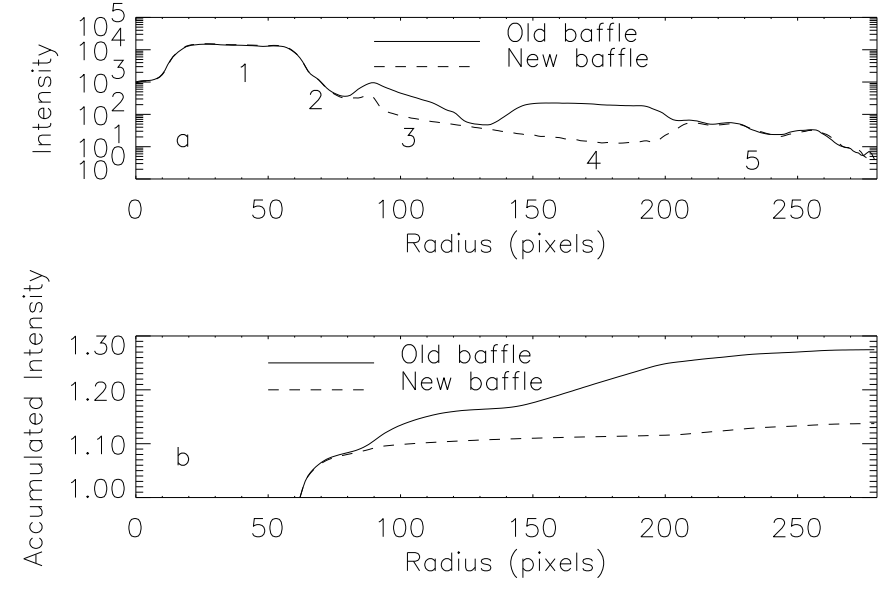

Fig. 4. The distribution of light in the pinhole images before and after the modification. a) The radially averaged profile for the two pinhole images. Note that the $y$-axis scaling is logarithmic. The numbers corresponds to the areas labelled in Fig. 1a. b) The intensity summed from the secondary mirror to radius $r$. The plot has been normalized by setting the total amount of light from the secondary mirror equal to 1 . It is evident from the figure that after the baffle modification most of the scattered light comes from the region close to the secondary mirror

The baffle modification consists of a tube with rings (annuli) inserted, which is mounted in the existing primary mirror baffle. The widths of the rings are such that an unvignetted field of 23 arcmin is available at the Cassegrain focus. Figure 1 shows the telescope baffles before (left) and after (right) the modification.

Compared to using a normal photographic camera the advantages of this approach are that the test is simple to do, and the images are standard CCD images thus allowing easy analysis; furthermore they are obtained with almost the same configuration as the one used when the science objects are observed. A pinhole camera also has the advantage of an easily changeable field of view and that objects are imaged in focus independent of their distance.

\subsection{Design and use of the camera}

The diameter of the pinhole should be a few tenths of a $\mathrm{mm}$, in order to obtain a proper spatial resolution. The opaque plate must be as thin as possible compared to the diameter of the pinhole. Otherwise vignetting at large angles to the axis of symmetry will occur. The pinhole plate should be placed at a distance from the CCD corresponding to 1 to 2 CCD diameters, to get an appropriate field of view. Care should be taken to seal any other passages for light than the pinhole.

Preparation of the images for analysis consists of bias and background subtraction. The background originates from light bypassing the pinhole, e.g., due to construction flaws in the filter housing. A constant background level may be estimated from an assumed dark area in the image, or a background exposure may be made with the pinhole covered.

\section{Results and discussion}

In Fig. 2 we show the obtained pinhole images. Figure 2a shows a pinhole image before the baffle was modified. It is obvious that a large fraction of the light in the image comes from regions outside the secondary mirror. The numbers in Fig. 2a correspond to reflections from the following regions (also marked in Fig. 1a):

1. The secondary mirror

2. The inside of the secondary mirror baffle

3. The upper end of the primary mirror baffle. The dark area between this region and region 4 is caused by the widening of the primary baffle; see also Fig. 1

4. The lower end of the primary mirror baffle

5. A metal 'collar' located in the Cassegrain hole. The dark areas are caused by holes drilled in the collar.

Figure 3 shows a surface plot of the pinhole image. This makes it easy to see the very asymmetric distribution of light outside the secondary mirror. It is this asymmetry which causes the observed flat field to depend on the position of the instrument rotator.

Figure $2 \mathrm{~b}$ shows the resulting pinhole image after modification of the baffle; note the large reduction in light outside the secondary mirror. This is also clearly seen in the surface plot in Fig. 3; apart from reducing the total amount of scattered light, the baffle has led to a more uniform distribution of the remaining scattered light.

A quantitative estimate of the improvement can be found in Fig. 4. Radially averaged profiles are shown in Fig. 4a, and the accumulated light as a function of radius in Fig. 4b. From the figure it is evident that a 2-fold reduction in the amount of scattered light has been achieved. It is also evident that a further reduction in scattered light can be achieved by improving the baffling close to the secondary mirror. This should reduce it below $\sim 5 \%$ of the amount of light 'contained' in the image of the secondary mirror. A small amount of light is still reflected off the 'collar' in the Cassegrain hole. Due to the large angle to the optical axis, this will very likely introduce uneven illumination of the CCD. Recently the baffle modification has been extended to include the collar.

We can also estimate the improvement of 'standard' dome- and sky flats. By creating quotients of dome and sky flat fields obtained at various rotator angles the quality of the flat fields can be evaluated (but this method cannot detect a constant or circular symmetric component of the scattered light). Before installation of the improved baffle we found a typical variation of $1 \%$ per arc minute. After installation this figure improved to slightly less than $0.2 \%$ per arc minute. The total field of the camera is $3.7 \times 3.7$. 


\section{Conclusion}

We have presented a simple method to test for the presence of scattered light in telescopes. By placing a pinhole mask in front of a CCD, a camera obscura is created. Images obtained in this configuration show the inside of the telescope and any source of light in the field of view, other than the secondary mirror. The test can be done in a few hours at any telescope.

For telescopes without a field de-rotator, or instrument rotator, it may be especially valuable since flat fields are always collected with the CCD in the same position relative to the telescope, and thus it may hide an asymmetric component of scattered light. However with the pinhole camera such a component would show up easily.

At the Nordic Optical Telescope the amount of scattered light reaching the CCD was reduced by a factor of 2 by improving the existing primary mirror baffle. Moreover this caused the remaining scattered light to be symmetric. This has virtually eliminated the dependence on instrument rotator position of both dome and sky flat fields.

Acknowledgements. During this work of identifying the scattered light at NOT we have enjoyed discussions with Bjarne
Thomsen, Jens Hjorth, Hans Kjeldsen, and Michael Andersen. ANS thanks the NOT staff for help in producing the baffle modification. FG acknowledges financial support from the Danish Board for Astronomical Research.

\section{References}

Andersen M.I., Freyhammer L., Storm J., in procedings of Calibrating and understanding ESO and HST instruments, Garching, April 1995

Garnavich P.M., Vandenberg D.A., Zurek D., Hesser J.E., 1994, AJ 107, 1097

Kuhn J.B., Lin H., Loranz D., 1991, PASP 103, 1097

Penny A., Griffiths B., RGO Gemini Newslett. No. 33, p. 4

Stetson P.B., Harris W.E., 1988, AJ 96, 909

Tobin W, in Stellar Photometry - Current Techniques and Future Developments, IAU Colloquium No. 136, Dublin, Ireland, August 1992

Kjeldsen H., Frandsen S., 1992, PASP 104, 413

Gilliland R.L., Brown T.M., Kjeldsen H., et al., 1993, AJ 106, 244 